\title{
Gleason Score as Predictor of Clinicopathologic Findings and Biochemical (PSA) Progression Following Radical Prostatectomy
}

\author{
Marbele S. Guimaraes, Maisa M. Quintal, Luciana R. Meirelles, Luis A. Magna, Ubirajara \\ Ferreira, Athanase Billis
}

Departments of Anatomic Pathology (MSG, MMQ, LRM, AB), Medical Genetics and Biostatistics (LAM), and Urology (UF), School of Medicine, State University of Campinas (Unicamp), Campinas, SP, Brazil

\begin{abstract}
Objective: There is evidence showing that Gleason grading of prostatic adenocarcinoma is one of the most powerful predictors of biological behavior and one of the most influential factors used to determine treatment for prostate cancer. The aim of the current study was to compare the Gleason score for needle biopsy to the Gleason score for the correspondent surgical specimen, find any possible difference in the biochemical (PSA) progression following surgery in upgraded cases, correlate Gleason score in the specimens to several clinicopathologic variables, and compare outcomes between patients with low-grade vs. high-grade Gleason and Gleason scores 3+4 vs. $4+3$.

Materials and Methods: The study population consisted of 200 consecutive patients submitted to radical prostatectomy. Biochemical progression was defined as PSA $\geq 0.2 \mathrm{ng} / \mathrm{mL}$. Time to PSA progression was studied using the Kaplan-Meier product-limit analysis.

Results: In $47.1 \%$ of the cases, there was an exact correlation and $40.6 \%$ of cases were underestimated in the biopsies. Half of the tumors graded Gleason 6 at biopsy were Gleason score 7 at surgery. These upgraded tumors had outcomes similar to tumors with Gleason score 7 in both biopsy and surgery. There was a positive correlation of high-grade Gleason score in the surgical specimens to higher preoperative PSA, more extensive tumors, positive margins and more advanced pathologic staging. Tumors with a Gleason score $\geq 7$ have lower PSA progression-free survival vs. Gleason scores $<7$. In this series, there was no significant difference when comparing Gleason scores of $3+4$ vs. $4+3$.

Conclusions: The findings support the importance of Gleason grading for nomograms, which are used by clinicians to counsel individual patients and help them make important decisions regarding their disease.
\end{abstract}

Key words: prostatic neoplasms; prostatectomy; Gleason grading system

Int Braz J Urol. 2008; 34: 23-9

\section{INTRODUCTION}

There is evidence showing that a Gleason grading of prostatic adenocarcinoma is one of the most powerful predictors of biological behavior and one of the most influential factors used to determine treatment for prostate cancer. The Gleason grading system is the most commonly used grading system for 
prostate cancer in the United States and is gaining worldwide acceptance. There are several unique aspects of the Gleason grading system including the fact that is solely based on the architectural pattern and that cytologic features are not factored in (1-3).

The aim of the current study report was: a) to compare the Gleason score on needle biopsy to the Gleason score in the correspondent surgical specimen; b) to find any possible difference in the biochemical (PSA) progression following surgery in upgraded cases; c) to correlate the Gleason score in the surgical specimen with the variables: preoperative serum PSA, tumor extent, positive surgical margins, and extraprostatic extension (pT3a and/or pT3b); and, d) to compare biochemical (PSA) progression following surgery between patients with Gleason low-grade vs. high-grade, and Gleason score $3+4$ vs. $4+3$.

\section{MATERIAL AND METHODS}

This retrospective study was based on 200 consecutive patients submitted to retropubic radical prostatectomy from January 1997 to July 2004 at our Institution. The Gleason score on all needle biopsies (58.9\% sextant biopsies and $41.1 \%$ extended biopsies) was compared to the Gleason score on the correspondent surgical specimen by one senior pathologist $(\mathrm{AB})$. The mean and median cores for the extended biopsies were 9.3 and 8.5 respectively. The Gleason score in the specimen was evaluated to find any association with the variables: preoperative serum PSA, tumor extent, positive margins, extraprostatic extension (pT3a and / or pT3b), and biochemical (PSA) progression following surgery.

The previously fixed surgical specimen was weighed, measured and the entire surface inked. The bladder neck and apical margins were amputated. From each cone-shaped amputated margin, 8 fragments were processed through perpendicular sections relative to the margins. The rest of the prostate was serially cut in transverse sections at 3 to $5 \mathrm{~mm}$ intervals. The prostate slices were subdivided into quadrants and labeled to allow for reconstruction as wholemount sections. Blocks were embedded in paraffin, cut at $6 \mu \mathrm{m}$, and one section from each block was stained with hematoxylin and eosin.

Presence of adenocarcinoma was diagnosed according to the criteria of Mostofi \& Price (1). The diagnosis was based on invasion or architectural disturbance. Histological grading was performed according to the Gleason system (2). Prostatic carcinomas with final score $<7$ were considered low-grade; and, with final score $\geq 7$ were considered high-grade (3). Extraprostatic extension was diagnosed according to Bostwick \& Montironi (4), whenever cancer was seen in adipose tissue, and corresponds to pT3a in the 2002 TNM staging system (5). Seminal vesicle invasion (pT3b) was defined as an invasion of the muscular wall, as described by Epstein et al. (6). Tumor extent was estimated by use of a point-count method (7). Drawn on a sheet of paper, each quadrant of the whole mount sections contained 8 equidistant points. During the microscopic examination of the slides, the tumor area was drawn on the correspondent quadrant seen on the paper. At the end of the examination, the amount of positive points represented an estimate of the tumor extent.

The data were analyzed using the MannWhitney test for comparison of independent samples and Spearman's coefficient of rank correlation to evaluate the association of Gleason score with preoperative serum PSA and tumor extent. Time to biochemical (PSA) progression-free survival was studied using the Kaplan-Meier product limit analysis; the comparison between the groups was done using the log-rank test. Biochemical progression was defined as PSA $\geq 0.2 \mathrm{ng} / \mathrm{mL}$ according to recent recommendation of the American Urological Association (8). P value $<0.05$ was considered statistically significant. All statistical analyses were performed using Statistica 5.5 (StatSoft, Inc., Tulsa, OK, USA).

\section{RESULTS}

We compared the Gleason score on 187 needle biopsies with matched whole mounted radical retropubic prostatectomy specimens. Twelve patients without information about Gleason grade on needle biopsy were excluded, as well as one patient without 
carcinoma in the prostatectomy specimen. There was exact correlation in $47.1 \%$ of cases. The Gleason grade was higher (underestimated in the biopsies) in $40.6 \%$ of cases and lower (overestimated in the biopsies) in $12.3 \%$ of cases. The distribution in biopsies was $2.1 \%, 68.1 \%, 24.5 \%$ and $5.3 \%$ of cases for Gleason score 2-5, 6, 7, and 8-10 respectively; in the surgical specimens, distribution was $8.5 \%, 27.6 \%$, $57.3 \%$, and $6.5 \%$ for the same Gleason scores respectively. From a total of 128 tumors with a Gleason score 6 in the biopsy, 65/128 (50.8\%) tumors were Gleason score 7 in the surgical specimen. From a total of 46 tumors with Gleason score 7 in the biopsy, $36 / 46(78.3 \%)$ tumors were also 7 in the surgical specimen.

The data were analyzed using Spearman's coefficient of rank correlation to evaluate the association of Gleason score in the surgical specimen with preoperative serum PSA in 199 patients. One case showing no carcinoma in the prostatectomy specimen was excluded. The median value of preoperative PSA was $10.46 \mathrm{ng} / \mathrm{mL}$ (range: 0.28 to $50 \mathrm{ng} / \mathrm{mL}$ ). There was a positive correlation, albeit weak, of high-grade Gleason score in the surgical specimens to higher preoperative serum PSA $(\mathrm{p}=0.032 ; \mathrm{r}=0.152)$.

The tumor extent in the surgical specimens ranged from 1 to 225 positive points (mean 37.5 positive points). Twenty-eight cases were excluded from the analysis because the point count method was not conducted, including one patient without carcinoma in the prostatectomy specimen. More extensive tumors correlated with high-grade Gleason score in the surgical specimens using Spearman's coefficient of rank correlation $(\mathrm{p}<0.001 ; \mathrm{r}=0.524)$.

A total of $82(41.2 \%)$ surgical specimens showed positive margins and $54(27.1 \%)$ extraprostatic extension (pT3a and/or pT3b). There was statistically significant correlation between high-grade Gleason score and positive margins $(\mathrm{p}<0.001)$ as well as extraprostatic extension ( $\mathrm{p}<0.001)$ using the Mann Whitney test. Only one case was excluded for this analysis. The higher percentage of positive margins (41.2\%) versus extraprostatic extension (27.1\%) may have occurred because in surgical specimens with positive margins but no extraprostatic extension the resulting stage is pT2+ or pT2x according to Epstein
(9). This stage denotes that elsewhere the tumor is organ confined, yet one cannot determine whether there is extraprostatic extension in the region of incision into the prostate because the edge of the prostate has been left in the patient.

For the analysis of time to biochemical progression, 14 patients without tests for serum PSA level following radical prostatectomy were excluded. The mean and median follow-up of our study (111 censored and 75 uncensored patients) was 30.3 and 25.5 months, respectively (range 1 to 99 months). During the follow-up 75/186 (40.3\%) patients developed biochemical (PSA) progression.

Time of biochemical (PSA) progression-free survival comparing 68 patients with Gleason score < 7 (low-grade) vs. 118 patients with Gleason score $\geq 7$ (high-grade) in the surgical specimens was statistically significant (log-rank, $\mathrm{p}=0.005)$ (Figure-1). At 5 years, the PSA progression-free survival rates were $73 \%$ and $35 \%$ for patients with low-grade and highgrade Gleason score in the surgical specimens, respectively.

Time of biochemical (PSA) progression-free survival comparing 87 patients with Gleason score $3+4=7$ vs. 19 patients with Gleason score $4+3=7$ was not statistically significant (log-rank, $p=0.193$ ) (Figure-2). At 5 years, the PSA progression-free survival rates were $37 \%$ and $32 \%$ for patients with Gleason score $3+4=7$ and Gleason score $4+3=7$, respectively.

\section{COMMENTS}

There have been several studies correlating core biopsy and radical prostatectomy Gleason grade (10-13). Bostwick (12) found for Gleason score an exact correlation in $35 \%$ of cases; in $40 \%$ of the cases the score was higher; and, in $25 \%$ was lower in the surgical specimen. Spires et al. (11) found an exact agreement between biopsy and excision in 58\%, higher grade in $37.5 \%$ and lower grade in $4.5 \%$ of the cases. Steinberg et al. (10) at the Johns Hopkins Hospital found an exact agreement of 58\%. In our study the exact agreement of Gleason score was $47.1 \%$. Gleason score was higher (underestimated on the biopsies) in $40.6 \%$ of cases and lower (overestimated 


\section{Cumulative Proportion Surviving (Kaplan-Meier)}

- Complete + Censored

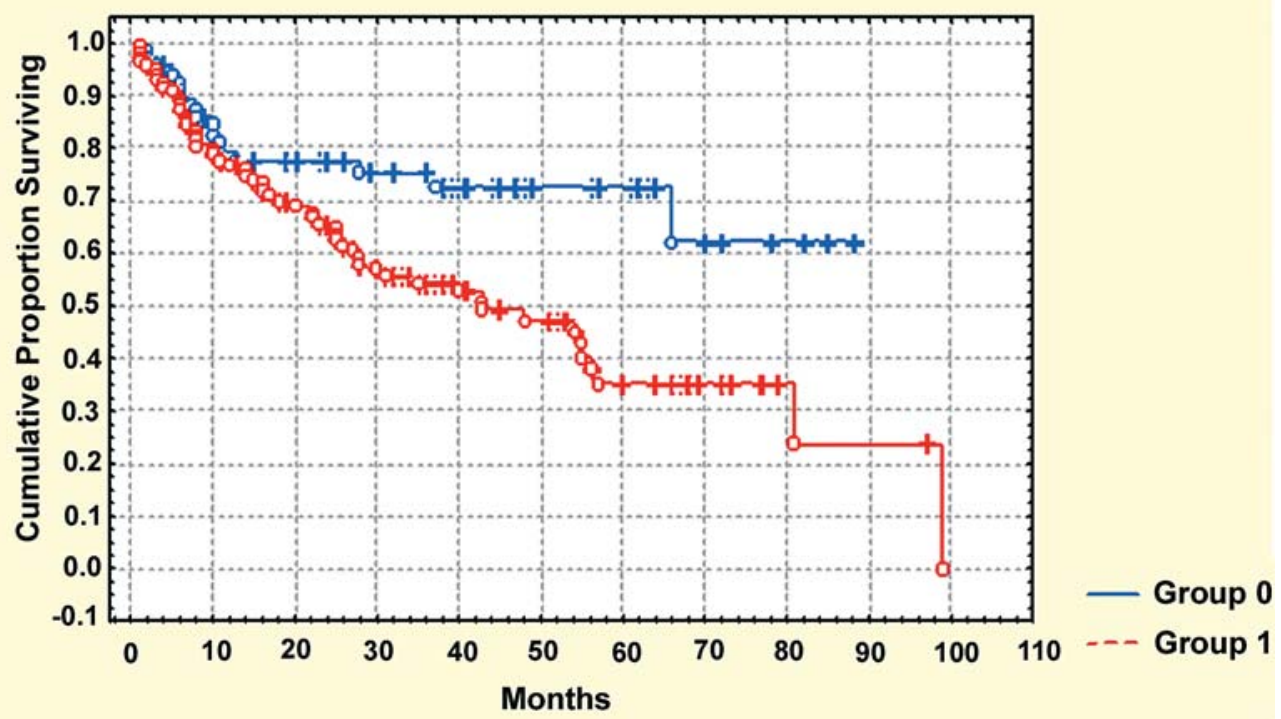

Figure 1 - Time to biochemical (PSA) progression-free survival comparing low-grade (group 0) vs. high-grade (group 1) Gleason score in the surgical specimen. Kaplan-Meier product-limit analysis.

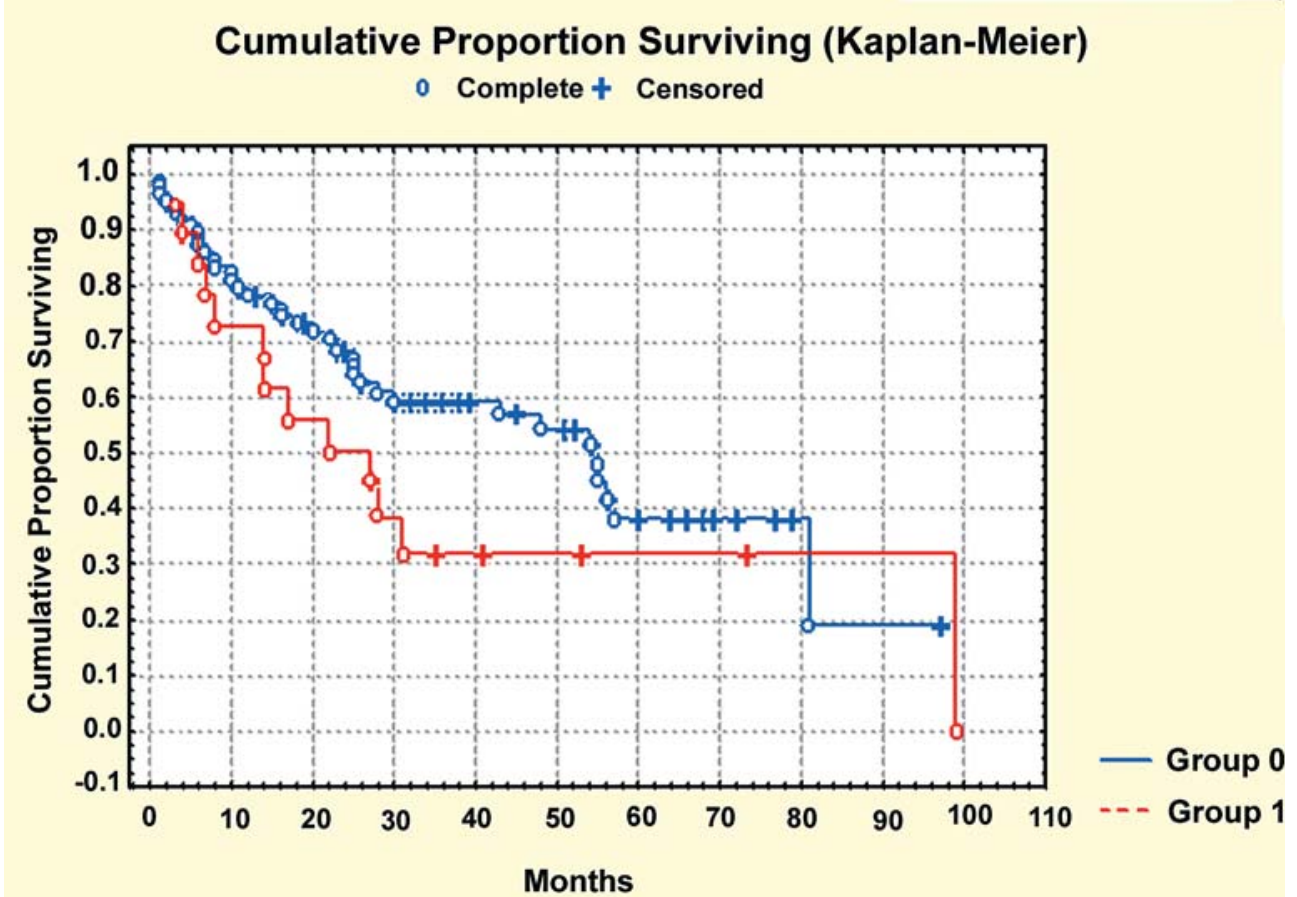

Figure 2 - Time to biochemical (PSA) progression-free survival comparing Gleason 3+4 (group 0) vs. Gleason 4+3 (group 1) in the surgical specimen. Kaplan-Meier product-limit analysis. 
on the biopsies) in $12.3 \%$ of cases. In our series, half of tumors graded Gleason score 6 at biopsy were Gleason score 7 at surgery. This finding is similar to the study by Pinthus et al. (13). We also compared the PSA progression-free survival of tumors that were scored 6 on prostate biopsy but were 7 on subsequent radical prostatectomy to those in tumors with a consistent rating of Gleason score 7 at biopsy and surgery. Upgraded Gleason score 6 to 7 tumors had outcome similar to those of Gleason score 7 in both biopsy and surgical specimen (log-rank, $p=0.359)$. This finding is also similar to the study by Pinthus et al. (13).

For a low-grade Gleason score in biopsies, urologists should consider the likelihood of tumor upgrading when contemplating treatments. High-grade Gleason score in biopsies have a high predictive value, which is enhanced when combined with other clinical parameters (14). Nomograms typically include the Gleason score in the pretreatment variables. Based on statistical modeling of cumulative, prospectively accrued data from a large consecutive series of patients, the nomograms have a reasonable discriminatory ability to counsel the urologist for pretreatment bone scans, magnetic resonance imaging for seminal vesicle involvement, and for lymph node dissection.

In general, serum PSA levels correlate with larger tumor volume, advanced pathologic stages, and higher grades. Although higher grade cancer produces less PSA per cell as compared to lower grade tumors, overall, poorly differentiated tumors are associated with higher PSA levels as these tumors tend to be larger and of more advanced stage (15). In our study, there was a positive correlation, albeit weak, of highgrade Gleason score in the surgical specimens to higher preoperative serum PSA.

Many studies have been published that consistently demonstrate the independent value of biopsy Gleason score in predicting pathologic stage at radical prostatectomy $(16,17)$. Based on these studies of needle biopsies and radical prostatectomy specimens, several groups have developed nomograms for predicting pathologic stage based on clinical stage, serum prostate-specific antigen (PSA), and needle biopsy Gleason score (18). The power of Gleason score in predicting outcome after radical prostatectomy has been repeatedly demonstrated in numerous reports $(19,20)$. Nomograms using the radical prostatectomy Gleason score and other variables are widely available. In patients with organ-confined, margin-negative tumors, the data are particularly compelling. Patients with Gleason score 6 tumors infrequently fail (21). Clinicians can use these nomograms to counsel individual patients and help them make important decisions regarding their disease. The findings of our study support the importance of Gleason grading in these nomograms. There was a statistically significant correlation between high-grade Gleason score and positive margins $(\mathrm{p}<0.001)$ as well as extraprostatic extension ( $\mathrm{p}<0.001)$ using the MannWhitney test.

During the follow-up 75/186 (40.3\%) patients developed biochemical (PSA) progression. This relatively high biochemical recurrence rate for our series may be due to considering a prostate specific antigen of $>$ or $=0.2 \mathrm{ng} / \mathrm{mL}$ as a biochemical recurrence, based on recent recommendations from the American Urological Association Prostate Guidelines (8). According to the Panel, strict definitions for biochemical recurrence are necessary to identify men at risk, but not necessarily to identify disease progression. The purpose for establishing this standard is for data reporting purposes. It is not intended to represent threshold value for initiating treatment. The Panel acknowledges that the clinical decision to initiate treatment will be dependent on multiple factors.

Time of biochemical (PSA) progression-free survival comparing patients with Gleason score $<7$ (low-grade) vs. Gleason score $\geq 7$ (high-grade) was statistically significant (log-rank, $p=0.005)$. At 5 years, the PSA progression-free survival rates were $73 \%$ and $35 \%$ for patients with low-grade and high-grade Gleason score, respectively. These PSA progressionfree survival rates were independent of margin status. Considering only patients with negative margins, the biochemical progression-free survival was $41 \%$ and $81 \%$ for high-grade and low-grade Gleason score, respectively (log-rank, $p=0.019$ ). For patients with prostate cancer, the Gleason score remains one of the most important parameters, affecting all aspects of their disease to include, potentially, the therapeutic stratification or approach. The importance placed by 
clinicians and patients on the many nomograms available cannot be overemphasized.

Tumors with a Gleason score of 7 have a significantly worse prognosis than those with a Gleason score of 6. Given the adverse prognosis associated with Gleason pattern 4, one would expect that whether a tumor is Gleason score $3+4$ or $4+3$ would influence prognosis (9). This issue has been controversial in the literature. No significant survival advantage was reported for Gleason pattern $3+4$ over $4+3$ by Oefelein et al. (22). Other investigators have shown that Gleason score $4+3$ has a worse prognosis than Gleason score $3+4(23,24)$. Chan et al. $(24)$ found that the 5year actuarial risk of progression was $15 \%$ and $40 \%$ for Gleason score 3+4 and 4+3 tumors respectively. In our study, time of biochemical (PSA) progressionfree survival comparing patients with Gleason score $3+4=7$ vs. Gleason score $4+3=7$ was not statistically significant (log-rank, $p=0.193)$. At 5 years, the PSA progression-free survival rates were $37 \%$ and $32 \%$ for patients with Gleason score $3+4=7$ and Gleason score $4+3=7$, respectively. We must consider, however, that this series has small numbers and short follow-up for any definitive conclusion to be drawn.

\section{CONCLUSIONS}

High-grade Gleason score tumors in the surgical specimens show a positive correlation with higher preoperative serum PSA, more extensive tumors, positive margins, and more advanced pathologic staging. Most of the patients show either an exact Gleason score or a higher score in the surgical specimen in comparison to the biopsy. Half of tumors graded Gleason score 6 at biopsy were Gleason score 7 at surgery. Time of biochemical (PSA) progression-free survival following surgery is significantly shorter for patients with high-grade Gleason score in the surgical specimens. Upgraded Gleason score 6 tumors in the biopsy to 7 in surgery, have outcomes similar to those of tumors with Gleason score 7 in both biopsy and surgery. In our series, time of biochemical (PSA) progression-free survival between patients with Gleason score $3+4$ vs. $4+3$ was not statistically significant. The findings support the importance of Gleason grading for nomograms, which are used by clinicians to counsel individual patients and help them make important decisions regarding their disease. Thus, accurate Gleason grading is essential information in the pathology report.

\section{CONFLICT OF INTEREST}

None declared.

\section{REFERENCES}

1. Mostofi, FK, Price EB Jr: Tumors of the Male Genital System, Atlas of Tumor Pathology, Second Series, Fascicle 8. Washington DC, Armed Forces Institute of Pathology. 1973; pp. 202-17.

2. Gleason DF, Mellinger GT: Prediction of prognosis for prostatic adenocarcinoma by combined histological grading and clinical staging. J Urol. 1974; 111: 5864.

3. Gleason DF: Histologic grading of prostate cancer: a perspective. Hum Pathol. 1992; 23: 273-9.

4. Bostwick DG, Montironi R: Evaluating radical prostatectomy specimens: therapeutic and prognostic importance. Virchows Arch. 1997; 430: 1-16.

5. International Union Against Cancer: Prostate. In: Sobin LH, Wittekind Ch. (ed.), TNM Classification of Malignant Tumours, 6th ed. New York, Wiley-Liss. 2002; pp.184-7.

6. Epstein JI, Carmichael M, Walsh PC: Adenocarcinoma of the prostate invading the seminal vesicle: definition and relation of tumor volume, grade and margins of resection to prognosis. J Urol. 1993; 149: 1040-5.

7. Billis A, Magna LA, Ferreira U: Correlation between tumor extent in radical prostatectomies and preoperative PSA, histological grade, surgical margins, and extraprostatic extension: application of a new practical method for tumor extent evaluation. Int Braz J Urol. 2003; 29: 113-9; discussion 120.

8. Cookson MS, Aus G, Burnett AL, Canby-Hagino ED, D'Amico AV, Dmochowski RR: Variation in the definition of biochemical recurrence in patients treated for localized prostate cancer: the American Urological Association Prostate Guidelines for Localized Prostate Cancer Update Panel report and recommendations for a standard in the reporting of surgical outcomes. J Urol. 2007; 177: 540-5. 
9. Epstein JI, Amin M, Boccon-Gibod L, Egevad L, Humphrey PA, Mikuz G, et al.: Prognostic factors and reporting of prostate carcinoma in radical prostatectomy and pelvic lymphadenectomy specimens. Scand J Urol Nephrol Suppl. 2005; 216: 34-63.

10. Steinberg DM, Sauvageot J, Piantadosi S, Epstein JI: Correlation of prostate needle biopsy and radical prostatectomy Gleason grade in academic and community settings. Am J Surg Pathol. 1997; 21: 566-76.

11. Spires SE, Cibull ML, Wood DP Jr, Miller S, Spires SM, Banks ER: Gleason histologic grading in prostatic carcinoma. Correlation of 18-gauge core biopsy with prostatectomy. Arch Pathol Lab Med. 1994; 118: 705-8.

12. Bostwick DG: Gleason grading of prostatic needle biopsies. Correlation with grade in 316 matched prostatectomies. Am J Surg Pathol. 1994; 18: 796-803.

13. Pinthus JH, Witkos M, Fleshner NE, Sweet J, Evans A, Jewett MA, et al.: Prostate cancers scored as Gleason 6 on prostate biopsy are frequently Gleason 7 tumors at radical prostatectomy: implication on outcome. J Urol. 2006; 176: 979-84; discussion 984.

14. Amin M, Boccon-Gibod L, Egevad L, Epstein JI, Humphrey PA, Mikuz G, et al.: Prognostic and predictive factors and reporting of prostate carcinoma in prostate needle biopsy specimens. Scand J Urol Nephrol Suppl. 2005; 216: 20-33.

15. Partin AW, Carter HB, Chan DW, Epstein JI, Oesterling JE, Rock RC, et al.: Prostate specific antigen in the staging of localized prostate cancer: influence of tumor differentiation, tumor volume and benign hyperplasia. J Urol. 1990; 143: 747-52.

16. Partin AW, Kattan MW, Subong EN, Walsh PC, Wojno $\mathrm{KJ}$, Oesterling JE, et al.: Combination of prostate-specific antigen, clinical stage, and Gleason score to predict pathological stage of localized prostate cancer. A multi-institutional update. JAMA. 1997; 277: 1445-51. Erratum in: JAMA 1997; 278: 118.
17. Partin AW, Mangold LA, Lamm DM, Walsh PC, Epstein JI, Pearson JD: Contemporary update of prostate cancer staging nomograms (Partin Tables) for the new millennium. Urology. 2001; 58: 843-8.

18. Han M, Partin AW: Nomograms for clinically localized prostate cancer. Part I: radical prostatectomy. Semin Urol Oncol. 2002; 20: 123-30.

19. D'Amico AV, Whittington R, Malkowicz SB, Fondurulia J, Chen MH, Tomaszewski JE, et al.: The combination of preoperative prostate specific antigen and postoperative pathological findings to predict prostate specific antigen outcome in clinically localized prostate cancer. J Urol. 1998; 160: 2096-101.

20. Kattan MW, Wheeler TM, Scardino PT: Postoperative nomogram for disease recurrence after radical prostatectomy for prostate cancer. J Clin Oncol. 1999; 17: 1499-507.

21. Palisaar RJ, Graefen M, Karakiewicz PI, Hammerer PG, Huland E, Haese A, et al.: Assessment of clinical and pathologic characteristics predisposing to disease recurrence following radical prostatectomy in men with pathologically organ-confined prostate cancer. Eur Urol. 2002; 41: 155-61.

22. Oefelein MG, Smith ND, Grayhack JT, Schaeffer AJ, McVary KT: Long-term results of radical retropubic prostatectomy in men with high grade carcinoma of the prostate. J Urol. 1997; 158: 1460-5.

23. Han M, Partin AW, Pound CR, Epstein JI, Walsh PC: Long-term biochemical disease-free and cancer-specific survival following anatomic radical retropubic prostatectomy. The 15-year Johns Hopkins experience. Urol Clin North Am. 2001; 28: 555-65.

24. Chan TY, Partin AW, Walsh PC, Epstein JI: Prognostic significance of Gleason score 3+4 versus Gleason score $4+3$ tumor at radical prostatectomy. Urology. 2000; 56 : 823-7.
Accepted after revision:

September 1, 2007

\section{Correspondence address:}

Dr. Athanase Billis

Dep. de Anatomia Patológica, FCM

UNICAMP, Caixa Postal 6111

Campinas, SP, 13084-971, Brazil

Fax: + 55 193289-3897

E-mail: athanase@fcm.unicamp.br 